\title{
Estudio comparativo de la relación existente entre resistencia media y concentración en hormigones fabricados con portland $y$ portland con adiciones activas
}

\author{
ANTONIO GARRIDO HERNANDEZ \\ Director del Laboratorio del Colegio Oficial de Aparejadores y Arquitectos Técnicos de Murcia
}

\section{$R E S U M E N$}

Este trabajo pretende mostrar las diferencias encontradas entre la resistencia media a 28 dias obtenidas en laboratorio y la prevista cuando la relacion $C / A$ había sido calculada con la fórmula general de De la Peña. Esta diferencia se considera suficiente como para justificar la modificación del contenido de la fórmula, aunque no de su estructura. Se sugiere que la diferencia pueda deberse a la utilización de cemento PA, cuando la formula tenga su origen en la experimentacion con cemento portland puro. Si ello es asi, el tema quedaria reducido a una generalización de la formula a otro tipo de cemento.

\section{$S U M M A R Y$}

This study attempts to show the differences found between average resistence, after 28 days, obtained in the laboratory and that predicted when the $C / A$ relationship has been calculated using the general De la Peña formula. This difference is considered to be sufficient to justify modifying the content of the formula, though not that of its structure. It is suggested that the difference may be due to the use of PA cement, when the formula originates from the tests with pure portland cement. If this is the case, it would simply be a question of generalizing the formula to another type of cement.

\section{JUSTIFICACION}

En un trabajo de confección de tablas para dosificar con cemento PA realizado en el laboratorio del Colegio Oficial de Aparejadores y Arquitectos Técnicos de Murcia se observó que las resistencias medias obtenidas a los 28 días no alcanzaban las previstas cuando la relación $C / A$ había sido calculada con la fórmula general de De la Peña. Estas diferencias superaban lo que podrían considerarse desviaciones no previsibles propias de, por ejemplo, el cambio de la categoria real del cemento, sino que afectaban de modo más profundo al contenido de la mencionada fórmula. Para comprobarlo se analizó, primero la estructura de la fórmula, para, después proceder a su reconstrucción con los datos empíricos en nuestro poder.

\section{ANALISIS DE LA FORMULA DE DE LA PEÑA}

Es conocido, empiricamente, que la resistencia media de un hormigón es proporcional a la concentración o relación $C / A$ del mismo. Una fórmula que expresa esta relación es la muy conocida de De la Peña (1):

$$
C / A=R_{\mathrm{m}} \cdot K+0.5
$$


siendo:

$C / A=$ Relación cemento-agua en peso.

$R_{\mathrm{m}}=$ Resistencia media del hormigón en $\mathrm{kp} / \mathrm{cm}^{2}$.

$K=$ Un coeficiente que depende del tipo de árido y la categoria nominal del cemento.

Esta fórmula presenta la forma general algebraica siguiente: $Y=a X+b$ que es la de una recta de origen $(0, b)$ y pendiente $a$. Por tanto la fórmula de De la Peña representa la ecuación de una recta que relaciona la variable dependiente $(C / A)$ y la variable independiente $\left(R_{\mathrm{m}}\right)$.

La pendiente de esta recta viene dada por el coeficiente $K$ que, como queda dicho, es función del tipo de árido y la categoria del cemento.

De la Peña presenta el siguiente cuadro para los distintos valores de $K(2)$ :

CUADRO 1

Valores de $K$

\begin{tabular}{|c|c|c|}
\hline Conglomerante & Aridos rodados & Aridos machacados \\
\hline Categoria 250 & 0.0072 & 0.0046 \\
Categoria 350 & 0.0054 & 0.0035 \\
Categoria 450 & 0.0045 & 0.0030 \\
Categoria 550 & 0.0038 & 0.0026 \\
\hline
\end{tabular}

Como consecuencia la pendiente de la recta presenta distintos valores para hormigones, que claborados con un mismo tipo de árido (rodado o machacado), se dosifiquen con distintas categorias de cemento portland. (Figura 1).

La relación entre los valores de $K$ (pendiente de la ecuación general) y la categoria del cemento la proporcionan las siguientes ecuảciones (3).

$$
\begin{aligned}
& K=0,4 \cdot r+40 \text { (árido rodado) } \\
& K=0,6 \cdot r+60 \text { (árido machacado) }
\end{aligned}
$$

Estas ecuaciones son del mismo tipo que la ecuación general. Tanto una como la otra expresan, no relaciones lógicas, sino empiricas y son, por tanto rectas de regresión de una variable sobre

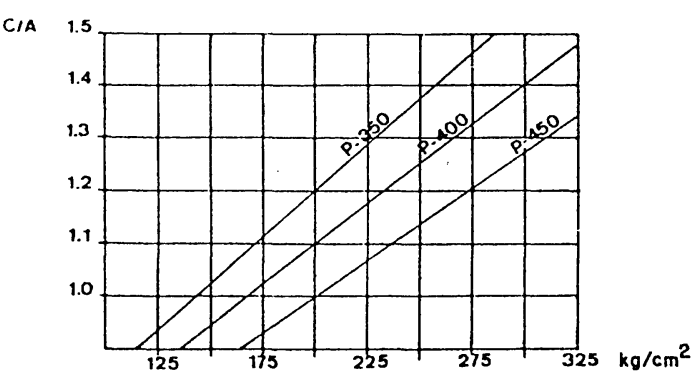

Fig. 1.- Rectas que representan la relación entre $C / A$ y $R_{\mathrm{m}}$ para distintas categorias del mismo tipo de cemento. otra. De este modo las relaciones entre:

- La concentración $C / A$ y resistencia media.

- La pendiente de la recta y la categoría del cemento se determinan mediante la elaboración de hormigones de referencia y variantes que midan la influencia de las variables.

\section{GENERALIZACION DE LA ECUACION GENERAL DE DE LA PEÑA}

Realizado el análisis de la ecuación de De la Peña del que se deduce su carácter de recta de 
regresión de la relación $C / A$ sobre la resistencia media se puede plantear su reconstrucción desde supuestos distintos si fuera necesario.

Las variables consideradas relevantes por De la Peña son:

- Categoria del cemento.

- Tipo de árido.

Pero todo ello en el marco general del cemento portland puro. Dado que el cemento habitualmente utilizado en la actualidad es PA-350, creemos que se puede intentar reconstruir la ecuación general con los datos de los trabajos realizados en el laboratorio del Colegio Oficial de Aparejadores y Arquitectos Técnicos de Murcia. Con ello se comprobaria si la ecuación se modifica y en qué cuantia lo haria con el nuevo tipo de cemento.

En estos trabajos la fórmula de partida fue la general de De la Peña en su aplicación para árido machacado y categoría nominal del cemento 350 .

$$
C / A=R_{\mathrm{m}} \cdot 0,0035+0.5
$$

Como se claboraron hormigones tipo $\mathrm{H}-150$ y H-175 y dos condiciones de ejecución previsibles resultan cuatro valores de resistencia media esperada.

$$
\begin{array}{llll}
H-150 \text { y } V=0,10 & R_{\mathrm{m}}=189 \mathrm{kp} / \mathrm{cm}^{2} & H-150 \text { y } V=0,20 & R_{\mathrm{m}}=243 \mathrm{kp} / \mathrm{cm}^{2} \\
H-175 \text { y } V=0,10 & R_{\mathrm{m}}=219 \mathrm{kp} / \mathrm{cm}^{2} & H-175 \text { y } V=0,20 & R_{\mathrm{m}}=280 \mathrm{kp} / \mathrm{cm}^{2}
\end{array}
$$

\begin{tabular}{|c|c|c|c|c|c|c|c|}
\hline $\begin{array}{c}\text { Relación } \\
\text { C/A }\end{array}$ & $\begin{array}{l}\text { Designación } \\
\text { hormigón }\end{array}$ & $\begin{array}{r}\text { Agua } \\
1 / \mathrm{m}^{3}\end{array}$ & $\begin{array}{c}\text { Cemento } \\
\mathrm{kg} / \mathrm{m}^{3}\end{array}$ & $\begin{array}{c}\text { Resistencia } \\
\text { S/UNE } 7240 \\
\text { y UNE } 7242 \\
\mathrm{kp} / \mathrm{cm}^{2}\end{array}$ & $\begin{array}{c}\text { Resistencia } \\
\text { media } \\
(1) \\
\mathrm{kp} / \mathrm{cm}^{2}\end{array}$ & $\begin{array}{c}\text { Resistencia } \\
\text { prevista } \\
(2) \\
\mathrm{kp} / \mathrm{cm}^{2}\end{array}$ & $\begin{array}{c}\text { Relación } \\
(2) /(1)\end{array}$ \\
\hline 1,16 & $\begin{array}{l}150 / \mathrm{P} / 40-0.10 \\
150 / \mathrm{B} / 40-0.10 \\
150 / \mathrm{P} / 20-0.10 \\
150 / \mathrm{B} / 20-0.10\end{array}$ & $\begin{array}{l}184 \\
198 \\
203 \\
211\end{array}$ & $\begin{array}{l}213 \\
230 \\
235 \\
244\end{array}$ & $\begin{array}{l}163 \\
166 \\
157 \\
162\end{array}$ & 162 & 189 & 1.166 \\
\hline 1.27 & $\begin{array}{l}175 / \mathrm{P} / 40-0.10 \\
175 / \mathrm{B} / 40-0.10 \\
175 / \mathrm{B} / 40-0.10 \\
175 / \mathrm{B} / 20-0.10\end{array}$ & $\begin{array}{l}184 \\
186 \\
209 \\
220\end{array}$ & $\begin{array}{l}233 \\
236 \\
266 \\
269\end{array}$ & $\begin{array}{l}174 \\
193 \\
196 \\
194\end{array}$ & 189 & 219 & 1.158 \\
\hline 1,35 & $\begin{array}{l}150 / \mathrm{P} / 40-0.20 \\
150 / \mathrm{P} / 20-0.20 \\
150 / \mathrm{B} / 40-0.20 \\
150 / \mathrm{B} / 20-0.20\end{array}$ & $\begin{array}{l}178 \\
197 \\
198 \\
211\end{array}$ & $\begin{array}{l}240 \\
266 \\
268 \\
285\end{array}$ & $\begin{array}{l}222 \\
230 \\
217 \\
222\end{array}$ & 217 & 243 & 1.119 \\
\hline 1.48 & $\begin{array}{l}175 / \mathrm{P} / 40-0.20 \\
175 / \mathrm{B} / 40-0.20 \\
175 / \mathrm{P} / 20-0.20 \\
175 / \mathrm{B} / 20-0.20\end{array}$ & $\begin{array}{l}178 \\
192 \\
197 \\
204\end{array}$ & $\begin{array}{l}264 \\
284 \\
291 \\
302\end{array}$ & $\begin{array}{l}251 \\
260 \\
278 \\
243\end{array}$ & 258 & 280 & $\left.1.0^{9}\right) 6$ \\
\hline
\end{tabular}

CUADRO 2 
Como se puede observar en ninguno de los casos de relación $C / A$ se ha alcanzado la resistencia media prevista. Cada valor de resistencia del cuadro corresponde a la media de dos parejas de probetas provenientes de dos amasadas distintas de cada designación de hormigón. La edad del ensayo fue de 28 dias.

Las bajas de resistencia respecto de la prevista disminuyen cuando aumenta la relación $C / A$. Se mueven entre el $9,6 \%$ y el $16,6 \%$, si a ello se añade que la categoría real del cemento empleado es $390 \mathrm{kp} / \mathrm{cm}^{2}$ ( $11 \%$ superior a la nominal), el error en la predicción de la resistencia en un ensayo previo puede llegar al $28 \%$ desde el $21 \%$. Esta diferencia es excesiva para dejarla sin explicación. Dado que la única diferencia entre el trabajo realizado y los supuestos de la fórmula general es el empleo de cemento PA en vez de portland puro, cabe atribuir, en principio a este factor las diferencias observadas. Si esto es así, debe existir otra relación entre la resistencia media y la relación $C / A$. Procedemos a buscarla.

\section{Recta de regresión: (Figura 2)}

Parámetros:

$$
\begin{aligned}
& C / A=1,315 \\
& f_{\mathrm{cm}}=206 \mathrm{kp} / \mathrm{cm}^{2} \\
& B=0,00328 \\
& C / A=0,0033 \cdot R_{\mathrm{m}}+0,64
\end{aligned}
$$

Siendo el coeficiente de correlación $R_{0}=0,998$.

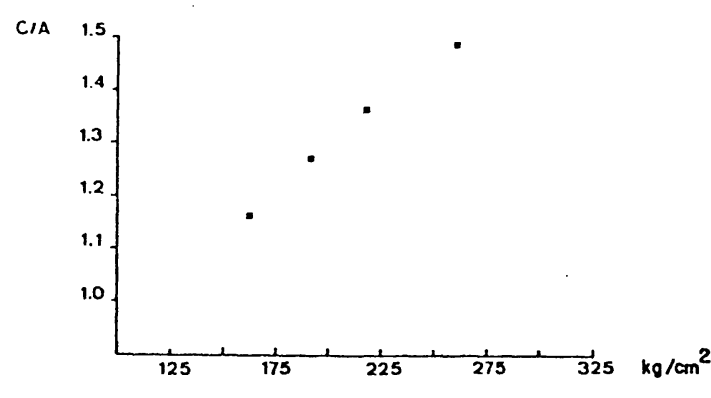

Fig. 2.-Diagrama de dispersión de las parejas $\left(R_{\mathrm{m}}, C / A\right)$.

La ecuación encontrada:

$$
C / A=0,0033 \cdot R_{\mathrm{m}}+0,64
$$

lo es para el cemento PA-350. Si como es razonable esperar, cuando cambie la categoria dentro del mismo tipo de cemento el valor 0,0035 será sustituido por otro. Con precisión, este valor, sería el propio de la categoría del cemento empleado $\left(390 \mathrm{kp} / \mathrm{cm}^{2}\right)$. Para completar, por tanto, el panorama con los cementos PA es preciso realizar ensayos que proporcionen los valores de la pendiente de la ecuación encontrada en su forma más general:

$$
C / A=K \cdot R_{\mathrm{m}}+0,64
$$

Se sugiere que para hacer más operativa la fórmula se encuentre la dispersión posible para el valor de $K$ correspondiente a una misma categoría nominal del cemento.

Por otra parte, vista la experiencia con el cemento PA, queda pendiente resolver el valor que tomaría el término independiente de la ecuación con otro tipo de cemento. Con lo visto se puede sugerir que la ecuación de De la Peña es un caso particular de una más general que tomaría la forma:

$$
C / A=K \cdot R_{\mathrm{m}}+J
$$

En la que $K$ depende del tipo de árido y la categoría real del cemento empleado y $J$ depende del tipo de cemento utilizado.

Por último mostramos en el gráfico de la figura 3 la diferencia entre la, que llamaremos ecuación del cemento P-350 y la del cemento PA-350. 
Fig. 3.-Representación gráfica de la ecuación que relaciona $C / A$ con $R_{\mathrm{m}}$ para el cemento PA-350 y $\mathrm{P}-350$.

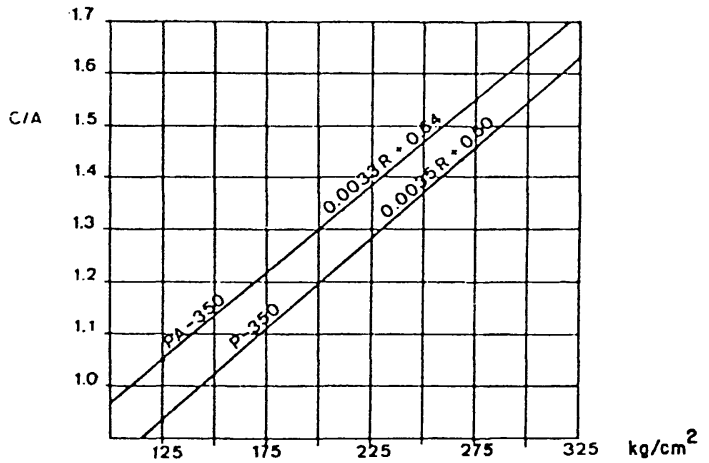

\section{REFERENCIAS BIBLIOGRAFICAS}

(1) Jimenez MONTOYA, P.; GARCiA MESEGUER, A. y MORAN CABRE, F.: 1981. Hormigón Armado. Edición 11. ${ }^{\text {a }}$ Madrid.

(2) ARREDONDO, F.: 1977. Dosificación de Hormigones. 5." Edición. Madrid.

(3) A.E.C.C.: Técnicos de control de obras de hormigón.

\section{NOTA:}

El autor es consciente de que la conclusión que se deriva de su trabajo es particular para un tipo de cemento determinado y que se requeriría una campaña de ensayos más amplia que permitiera géneralizar o no, a todos los PAs, lo que apunta el cemento utilizado en este trabajo. El presentar los resultados obtenidos tiene por finalidad explícita el invitar, a aquellos que pudiesen estar interesados en el problema planteado, a que contribuyan a su resolución.

\section{publicacion del i.e.t.c.c.}

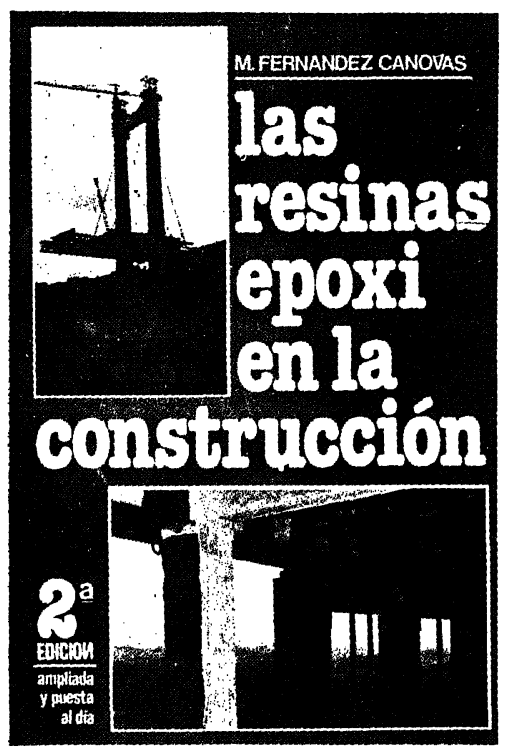

Manuel Fernández Cánovas Dr. Ingeniero de Construcción

Este libro, el primero en lengua castellana sobre resinas epoxi aplicadas a la construcción, está dirigido a arquitectos, ingenieros, constructores y aplicadores. En él, sobre una reducida base teórica imprescindible, se asienta toda una extensa gama de aplicaciones de gran interés.

El autor trabaja desde hace muchos años en el campo de la investigación, especialmente en el estudio de refuerzos y reparaciones estructurales realizados con resinas epoxi.

Con un lenguaje sencillo se tocan todos los problemas que pueden presentarse en la construcción y en los que la solución puede radicar en el correcto empleo de las resinas epoxi.

Se estudian los componentes de las formulaciones epoxi, sus propiedades fisicas y quimicas, y aplicaciones, deteniéndose, detalladamente, en las siguientes:

Unión de hormigón fresco a hormigón endurecido. --Unión de hormigones entre si.-Inyecciones de fisuras y grietas. -- Unión de acero a hormigón. -- Barnices y pinturas. - Las combinaciones brea-epoxi. - Revestimientos de depositos alimenticios. -..- Sellado de superficies cerámicas. - Protección de tubos. Los suelos epoxi en sus diferentes variantes. -- Terrazo epoxi. -.- Reparación de baches. - Reparación de despertoctos en estructuras. - Reparacion de carreteras de hormigón. - Juntas elásticas. - Guardacantos de tableros de puenes. - Refueres de pi vigas, forjados y zapatas, etc. - Consolidación de st

Se termina con unos capitulos dedicados a la limpieza y preparación de las superficies según los materiales a unir:

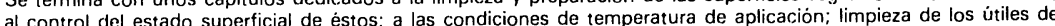
al control del estado superficial de éstos; a las condiciones de temperatura de aplicacion; limpieza de los útiles de métodos de ensayo de sistemas y aplicaciones epoxidicas.

Un volumen encuadernado en cartoné plastificado con lomo de tela, de $17 \times 24 \mathrm{~cm}$, compuesto de 334 páginas y 158 figuras y fotografias.

Madrid, 1981.

Precios: España, 1.700 ptas.; extranjero, \$ USA 34.00 